\title{
Towards an Exact Treatment of Exchange and Correlation in Materials: Application to the "CO Adsorption Puzzle" and Other Systems
}

\author{
Qing-Miao Hu, ${ }^{1, *}$ Karsten Reuter, ${ }^{1}$ and Matthias Scheffler ${ }^{1,2}$ \\ ${ }^{1}$ Fritz-Haber-Institut der Max-Planck-Gesellschaft, Faradayweg 4-6, D-14195 Berlin, Germany \\ ${ }^{2}$ Departments of Chemistry \& Biochemistry and Materials, UCSB, Santa Barbara, California 93106, USA
}

(Received 6 November 2006; published 23 April 2007)

\begin{abstract}
It is shown that the errors of present-day exchange-correlation (XC) functionals are rather short ranged. For extended systems, the correction can therefore be evaluated by analyzing properly chosen clusters and employing highest-quality quantum chemistry methods. The XC correction rapidly approaches a universal dependence with cluster size. The method is applicable to bulk systems as well as to defects in the bulk and at surfaces. It is demonstrated here for $\mathrm{CO}$ adsorption at transition-metal surfaces, where present-day $\mathrm{XC}$ functionals dramatically fail to predict the correct adsorption site, and for the crystal bulk cohesive energy.
\end{abstract}

DOI: 10.1103/PhysRevLett.98.176103

PACS numbers: 68.43.Bc, 71.15.Mb, 71.15.Nc

Electronic-structure theory is the base for a multiscale modeling of materials properties and functions (see, e.g., Ref. [1]). Obviously, if the needed accuracy is lacking at this base, there is little hope that accurate predictions can be made at any level of modeling that follows. For polyatomic systems, density-functional theory (DFT) with present-day exchange-correlation (XC) functionals has proven to be an excellent technique for calculations at this electronic-structure base. However, it is not as good for certain types of binding interactions. Accurate treatments of strong electronic correlations, van der Waals interactions, and molecular dynamics for electronically excited states represent unsolved challenges. Besides numerical approximations (e.g., the basis set, possible use of the pseudopotential approximation, etc.) that good theoretical work is typically scrutinizing, a satisfying test of the quality of the $\mathrm{XC}$ functional was not possible for bigger systems, so far. Sometimes the results obtained with different functionals have been compared, and, when they agreed, this was taken as an indicator for reliability. Though this was the best possible approach, it is neither safe nor justified. Exchange-correlation functionals are typically built on the homogeneous electron gas [the local-density approximation (LDA) [2]], adding corrections while ensuring consistency with known sum rules [e.g., the generalized gradient approximation (GGA) [3]], or they are constructed to reproduce certain data of some small molecules (e.g., the B3LYP functional [4]). There is no systematic expansion in terms of successively decreasing errors, and there is no proof that, e.g., the GGA will always work more trustfully than the LDA. On the other hand, for wave function methods, several promising concepts exist for better XC treatments also for extended systems (see Refs. [5,6], and references therein). However, these are not yet efficient, in particular, for metals.

It is often argued that the XC error is largely canceled when total-energy differences are studied and that the XC approximation affects the geometry only by a little.
However, this is generally not correct. A key example for the $\mathrm{XC}$ problem is the low-coverage adsorption of $\mathrm{CO}$ at the (111) surface of $\mathrm{Pt}$ or $\mathrm{Cu}$, where the LDA as well as the GGA predict that the molecule adsorbs in the threefoldcoordinated hollow site. Experiments, on the other hand, show undoubtedly that the adsorption site is in the onefoldcoordinated top site. Obviously, the conclusion based on DFT-LDA/GGA is even qualitatively incorrect, and, when comparing the calculated energies of the two sites in question, the error in the energy difference is indeed significant: In the LDA, it must be larger than $0.4 \mathrm{eV}$.

The comprehensive study of $\mathrm{CO}$ at $\mathrm{Pt}(111)$ by Feibelman et al. [7] set the ball rolling, showing that, when properly realizing all technical aspects of the calculations (e.g., basis set, supercell, cluster geometry), the LDA and GGA put the molecule at the wrong adsorption site. For several other close-packed transition-metal surfaces, including $\mathrm{Cu}(111)$, the situation is analogous (e.g., [8]). Shortly after the paper by Feibelman et al. [7], it was realized that the wrong site preference of $\mathrm{CO}$ may be related to the fact that DFT-LDA and GGA inaccurately describe the $\mathrm{CO}$ molecule's chemical bond. This was then expressed in terms of the highest occupied molecular orbital-lowest unoccupied molecular orbital splitting (the $5 \sigma$ and $2 \pi^{*}$ energy levels) or, correspondingly, the singlet-triplet excitation [9-13]. An upshift of the one electron $2 \pi^{*}$ level makes a partial charge transfer from the $5 \sigma$ to the $2 \pi^{*}$ orbital more difficult and, therefore, stabilizes the $\mathrm{CO}$ bond. Indeed, such a (semiempirical) upshift brought the $\mathrm{CO}$ molecule to the experimentally known site. Thus, the problem appears to be understood (to some degree), but altogether the situation is unsatisfactory. A first-principles theory should provide a reliable answer, and an add-on, which is triggered by a disagreement with experiment, questions the usefulness of the whole self-consistent procedure. We also note that the full correction of the LDA/GGA functional will not just shift the $\operatorname{CO} 2 \pi^{*}$ orbital to higher energies, it will also 
modify the substrate $d$ states and thereby the substrateadsorbate bonding in various terms. Below, we will take the "CO adsorption puzzle" as our main example for a systematic, nonempirical approach to correct XC errors. We will also discuss a fundamentally different case, namely, the bulk cohesive energy.

For small systems (say, up to 20-50 atoms), high-level quantum chemistry methods or the quantum Monte Carlo approach can be employed to obtain accurate results for the exchange-correlation energy. We will show that calculations on such small clusters are sufficient to evaluate the DFT-LDA error of extended systems. We will concentrate on $\mathrm{CO} / \mathrm{Cu}(111), \mathrm{CO} / \mathrm{Ag}(111)$, and $\mathrm{Cu}$ bulk as for these systems relativistic effects are small. We use accurate fullpotential augmented plane wave (LAPW/APW + lo) DFT calculations [14] with LDA [2] and GGA [3] functionals to calculate the $\mathrm{CO}$ adsorption energies with a numerical uncertainty of $\pm 0.02 \mathrm{eV}$ [15]. Supercells with relaxed, symmetric five-layer slabs are employed to model the low-coverage adsorption at $1 / 9$ monolayer on the extended surface. In good agreement with previous studies $[8,12,13]$, we obtain the fcc hollow site to be more stable than the correct top site (by $0.33 \mathrm{eV}$ within the LDA and by $0.11 \mathrm{eV}$ within the GGA).

Our approach works as follows (we are using here DFTLDA as the starting point, but, of course, one could as well start with other functionals, e.g., DFT-GGA): (i) Do supercell calculations for the extended system using DFT-LDA. (ii) Do cluster calculations with the same functional and same geometry as in step (i). It may be convenient to saturate the cluster surface by hydrogen adatoms, but there is, in principle, no need to do so. (iii) Do corresponding calculations for exactly the same cluster as in step (ii) but using an improved $\mathrm{XC}$ treatment. This may employ the B3LYP functional, Hartree-Fock (HF) plus Møller-Plesset perturbation theory (MP2), a coupled cluster, or a quantum Monte Carlo calculation.

The difference of the results of steps (iii) and (ii), i.e.,

$$
E^{\mathrm{XC} \text { corr. }}=E^{\text {cluster }}(\mathrm{XC}-\text { better })-E^{\text {cluster }}(\mathrm{LDA}),
$$

then is the XC correction. Why should this cluster quantity $E^{\mathrm{XCcorr}}$ be of much relevance for the extended system? Figure 1 shows how $E^{\text {XCorr. }}$ changes with cluster size using the $\mathrm{CO}$ adsorption energy in the fcc hollow site at $\mathrm{Cu}(111)$ as an example. These calculations $[16,17]$ were performed at the DFT-LDA, GGA, and B3LYP levels, as well as with HF-MP2. The correction of the LDA result is dramatic: It is of the order of several hundreds of $\mathrm{eV}$, and the different $\mathrm{XC}$ treatments all give noticeably different results. In this respect, we note that none of the employed methods (LDA, GGA, B3LYP, HF-MP2) fulfills the variational principle for the ground state of the true many-electron Hamiltonian; i.e., they all could, in principle, give results that are even below the true many-body ground state energy. For a free $\mathrm{CO}$ molecule, for example, the total energies are -3059.01 (LDA), -3079.83 (GGA), -3083.30 (B3LYP),

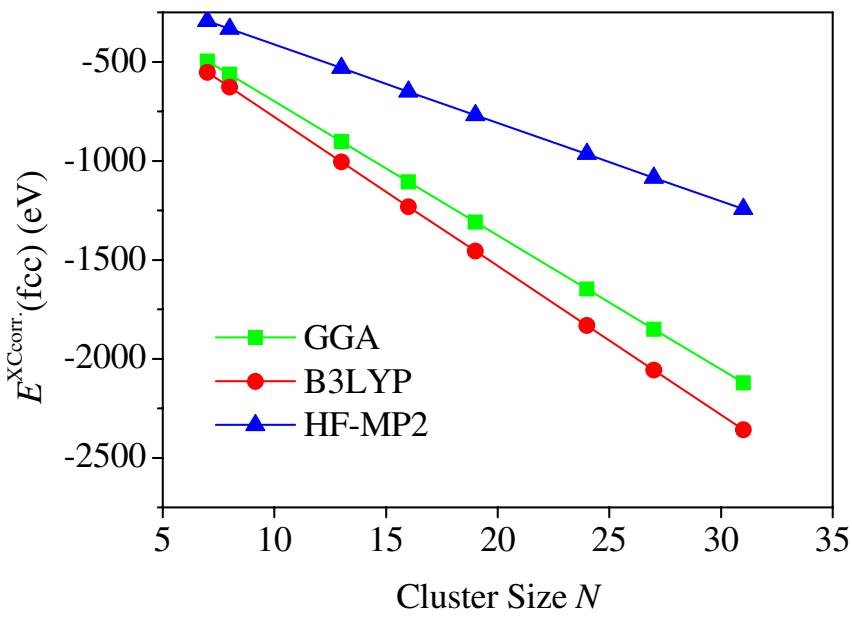

FIG. 1 (color online). Total-energy correction $E^{\text {XCcorr. }}$ [see Eq. (1)] with respect to the LDA as a function of cluster size and for $\mathrm{XC}=\mathrm{GGA}, \mathrm{B} 3 \mathrm{LYP}$, and HF-MP2 for the adsorption of $\mathrm{CO}$ at $\mathrm{Cu}(111)$ in the fcc hollow site.

and $-3075.89 \mathrm{eV}$ (HF-MP2), and the experimental value is $-3084.72 \mathrm{eV}$ [18]. Thus, the order is LDA $>$ HF-MP2 $>$ GGA $>$ B3LYP $>$ experiment, where the differences to the experimental values are between -25.7 (LDA) and $-1.4 \mathrm{eV}$ (B3LYP). The trend seen in Fig. 1 is thus the same as that of the free $\mathrm{CO}$ molecule.

Obviously, $E^{\mathrm{XCcorr}}$ is very different for different $\mathrm{XC}$ functionals. Interestingly, if we evaluate differences, e.g., for different adsorption sites, these differences rapidly approach a constant value. Figure 2 shows the difference of $E^{\mathrm{XCcorr}}$ for $\mathrm{CO}$ at $\mathrm{Cu}(111)$ in the fcc and in the top adsorption sites. Thus, XC-approximate total-energy differences of the extended surface can be corrected through

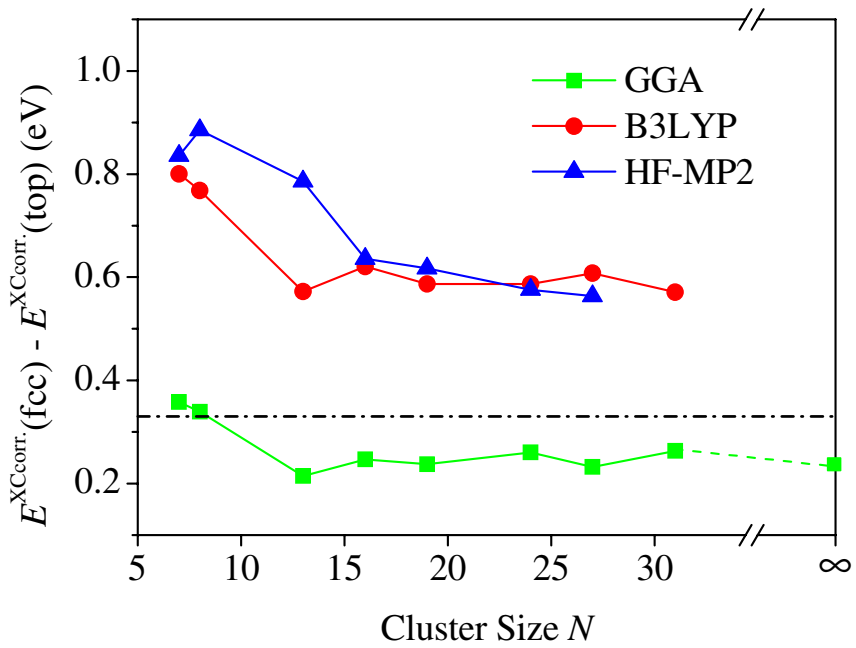

FIG. 2 (color online). The energy correction difference $E^{\text {XCcorr. }}$ (fcc) $-E^{\text {XCcorr. }}$ (top). The dashed-dotted line at $0.33 \mathrm{eV}$ marks the minimum $\mathrm{XC}$ correction required to obtain the correct top adsorption site. The $N=\infty$ result for the GGA-LDA correction was obtained by supercell calculations (LAPW/APW + lo). 
higher-level calculations for finite clusters, and the cluster size where the XC energy correction term $\Delta E^{\mathrm{XCcorr} .}$ becomes constant determines the efficiency of the local correction approach. For the GGA, we also have the supercell result at 1/9 monolayer coverage, where the adsorbateadsorbate interaction is negligible, and we added this $N=$ $\infty$ result as well. Figure 2 demonstrates that $\Delta E^{\mathrm{XCcorr}}$ is converged already for very small clusters (a $16 \mathrm{Cu}$ atom cluster appears to be sufficient). We emphasize that this applies to the differences and the LDA error. For adsorption or reaction energies, such clusters are by far too small. Apparently, the LDA error is even shorter ranged than Kohn's nearsightedness concept [19], which refers to interaction energies.

Using the converged values of $\Delta E^{\mathrm{XCcorr} .}$ enables us to correct the LDA energy of the slab calculations. The GGA correction decreases the wrong LDA preference for the fcc site, but it cannot yet change the energetic order of the hollow and top adsorption sites. However, at the B3LYP level, the top site is now more stable by $0.21 \pm 0.03 \mathrm{eV}$. Interestingly, an almost identical value of $0.28 \pm 0.04 \mathrm{eV}$ is obtained at the HF-MP2 level. This confirms the interpretation of earlier B3LYP studies $[11,20,21]$ that the main reason for the wrong site preference of LDA and GGA functionals is the self-interaction error (also present in the free $\mathrm{CO}$ molecule).

Table I gives the energies for all high-symmetry adsorption sites at the $\mathrm{Cu}(111)$ and $\mathrm{Ag}(111)$ surfaces, namely, top, bridge, fcc, and hcp. For $\mathrm{CO} / \mathrm{Cu}(111)$, the top site is now the most stable adsorption site, and the optimum diffusion energy barrier for the top-bridge-top pathway is $0.20 \mathrm{eV}$. For the other system, $\mathrm{CO}$ at $\mathrm{Ag}(111)$, already GGA yields the correct top site as the most stable one $[8,12]$, and it is interesting to verify that a higher-level XC treatment does not spoil this description. As shown in Table I, the energetic order is indeed not changed at the B3LYP level, and the top site is in fact further stabilized by $0.20 \mathrm{eV}$, so that the diffusion barrier for the top-bridge-top pathway is more than doubled: from 0.14 (GGA) to $0.35 \mathrm{eV}$ (B3LYP).

The approach is not just applicable to localized perturbations, such as an adsorbate or a defect. It can also be used to study the bulk cohesive energy. In this case, however, it is not possible to express the correction in terms of energy

TABLE I. Adsorption energies (in $\mathrm{eV}$ ) for low-coverage $\mathrm{CO}$ adsorption into different high-symmetry adsorption sites at $\mathrm{Cu}(111)$ and $\mathrm{Ag}(111)$. The values at the GGA and B3LYP levels are obtained through the $\mathrm{XC}$ energy correction scheme, using the LDA numbers as reference. The energy of the lowest energy structure is taken as energy zero.

\begin{tabular}{lccccc}
\hline \hline System & XC & Top & fcc & hcp & Bridge \\
\hline $\mathrm{Cu}(111)$ & GGA & +0.13 & +0.01 & 0 & +0.05 \\
& B3LYP & 0 & +0.21 & +0.26 & +0.20 \\
$\mathrm{Ag}(111)$ & GGA & 0 & +0.22 & +0.15 & +0.14 \\
& B3LYP & 0 & +0.42 & +0.43 & +0.35 \\
\hline \hline
\end{tabular}

differences so that cluster size and edge effects cancel. We therefore now write the total energy of the many-atom system as a sum over contributions assigned to the individual atoms: $E=\sum_{I} E_{I}$. Here $E_{I}$ is the energy contribution due to atom $I$. In the simplest, yet physically meaningful approach for metals, $E_{I}$ is roughly proportional to $\sqrt{c}$, where $c$ is the local coordination, i.e., the number of nearest neighbors (see, e.g., [22,23]). For our close-packed $\mathrm{Cu}$ clusters, we then get

$$
E=N * E^{\text {atom }}+\left(E^{\text {coh. }} / \sqrt{12}\right) \sum_{c=1}^{12} \sqrt{c} * N_{c},
$$

where $E^{\text {atom }}$ is the calculated total energy of the free atom at the given XC level, $N$ is the number of atoms in the cluster, $N_{c}$ is the number of atoms in the cluster that are $c$ fold-coordinated, and $E^{\text {coh. }}$ corresponds to the cohesive energy expected for an infinite size cluster. Figure 3 shows how $E^{\text {coh. }}$ changes with cluster size and how it depends on the XC functional.

It can be seen that the correction $\Delta E^{\text {coh. }}($ GGA-LDA $)=$ $E^{\text {coh. }}(\mathrm{GGA})-E^{\text {coh. }}$ (LDA), as well as those for the other $\mathrm{XC}$ functionals, is converged already for $N=24$ clusters with an uncertainty of $\pm 0.1 \mathrm{eV}$. Hartree-Fock shows the same convergence behavior as B3LYP but at a value of $-3.8 \mathrm{eV}$. For the GGA, we also performed calculations for the infinite crystal, and this gives the difference between the GGA and LDA cohesive energies as $-1.06 \mathrm{eV}$, in very close agreement to the converged value we get with our XC correction scheme. Neef and Doll [21] had obtained values of -1.05 and $-2.04 \mathrm{eV}$ for the (GGA-LDA) and the (B3LYP-LDA) corrections, respectively, which is rather close to our results given in Fig. 3. However, this (B3LYP-LDA) correction is too large to match experiment. The experimental cohesive energy for $\mathrm{Cu}$ is $3.49 \mathrm{eV}$ [24],

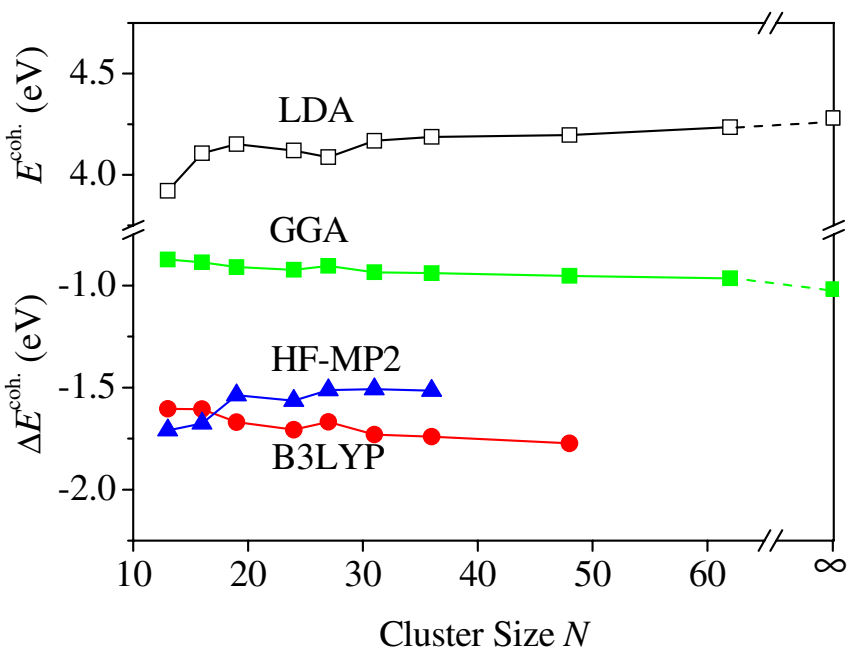

FIG. 3 (color online). Cohesive energy $E^{\text {coh. }}$ of $\mathrm{Cu}$ [cf. Eq. (2)] for the LDA (black line, open squares) and $\Delta E^{\text {coh. }}$ corrections with respect to the LDA for GGA, B3LYP, and HF-MP2. The $N=\infty$ result for the LDA and the GGA-LDA correction was obtained by crystal bulk calculations (LAPW/APW + lo). 
which differs by $-0.83 \mathrm{eV}$ from the LDA cohesive energy. Apparently, B3LYP is very bad in its description of $\mathrm{Cu}$ bulk. Also, our cohesive energy at the HF-MP2 level $(2.82 \pm 0.1 \mathrm{eV})$ is significantly smaller than the experimental value, but at this point we cannot rule out that the HF-MP2 convergence with cluster size is different from that of other treatments. In fact, whether or not HF-MP2 should work for metals needs a deeper theoretical analysis. A more detailed discussion will be given elsewhere [25].

All results in Fig. 3 were obtained for the LDA lattice constant. Of course, we could have easily optimized the lattice constants for the different treatments (or we could have shown the equations of state for the different XC levels). However, this would have complicated the graphs due to the intermingling of different effects. The focus of the present work is on metal surfaces and bulk. However, the methodology proposed in this Letter for correcting DFT-slab adsorption, cohesive, and diffusion energies is also applicable to semiconductors [e.g., $\mathrm{H}_{2}$ at $\mathrm{Si}(001)$ [26]] or ionic materials (e.g., $\mathrm{NaCl}$ ). Not surprisingly, here the efficiency of the $\triangle \mathrm{XC}$ approach is even better (for details, see [25]). The approach had been applied by Tuma and Sauer to protonation reactions in zeolites (combining DFT and HF-MP2) [27]. This work is interesting, as here the main source of error at the DFT level appears to be the lack of the long-range van der Waals tails, and by the correction scheme the authors could evaluate these van der Waals contributions to adsorption.

In summary, we presented a scheme to locally correct the total energy (or total-energy differences) for errors contained in present-day local or semilocal DFT functionals, e.g., the self-interaction and the lack of van der Waals interactions. When looking at appropriate energy differences, a smooth and rapid convergence of the XC correction with cluster size is observed. This enables us to reach convergence at very small cluster sizes or extrapolate to $N=\infty$. At these small cluster sizes that are treatable, e.g., with HF-MP2, it is only the XC correction, not the total energy, that is converged. The approach is demonstrated by computing the energetic order of the high-symmetry sites for the low-coverage adsorption of $\mathrm{CO}$ at $\mathrm{Cu}(111)$ and $\operatorname{Ag}(111)$. The corrections to potential-energy surfaces obtained with LDA or GGA are found to be significant: For $\mathrm{CO}$ diffusion at $\operatorname{Ag}(111)$, energy barriers are changed by more than a factor of 2 , and, for $\mathrm{CO}$ at $\mathrm{Cu}(111)$, even the topology is altered. Furthermore, the approach was applied to evaluate the cohesive energy of $\mathrm{Cu}$ bulk, enabling us to perform HF-MP2 calculations (via a systematic extrapolation, starting from the LDA) for an extended system. In this Letter, we addressed only changes in energy, but for forces the correction is straightforward, analogous to Eq. (1).

Discussions with Thorsten Kluener and Martin Fuchs are gratefully acknowledged. Part of the work was supported by the Deutsche Forschungsgemeinschaft (SFB658) and the Alexander von Humboldt Foundation.
*Permanent address: Institute of Metal Research, CAS, Shenyang 110016, People's Republic of China.

[1] K. Reuter, C. Stampfl, and M. Scheffler, in Handbook of Materials Modeling, edited by S. Yip (Springer, Berlin, 2005), Vol. 1, p. 149, ISBN 1-4020-3287-0.

[2] J. P. Perdew and Y. Wang, Phys. Rev. B 45, 13244 (1992).

[3] J. P. Perdew, K. Burke, and M. Ernzerhof, Phys. Rev. Lett. 77, 3865 (1996).

[4] A. D. Becke, J. Chem. Phys. 98, 5648 (1993).

[5] P. Fulde, Adv. Phys. 51, 909 (2002).

[6] B. Paulus, Phys. Rep. 428, 1 (2006).

[7] P. J. Feibelman et al., J. Phys. Chem. B 105, 4018 (2001).

[8] M. Gajdoš, A. Eichler, and J. Hafner, J. Phys. Condens. Matter 16, 1141 (2004).

[9] I. Grinberg, Y. Yourdshahyan, and A. M. Rappe, J. Chem. Phys. 117, 2264 (2002).

[10] R. A. Olsen, P.H. T. Philipsen, and E. J. Baerends, J. Chem. Phys. 119, 4522 (2003).

[11] A. Gil et al., Surf. Sci. 530, 71 (2003); G. Kresse, A. Gil, and P. Sautet, Phys. Rev. B 68, 073401 (2003).

[12] S. E. Mason, I. Grinberg, and A. M. Rappe, Phys. Rev. B 69, 161401(R) (2004).

[13] M. Gajdoš and J. Hafner, Surf. Sci. 590, 117 (2005).

[14] P. Blaha et al., WIEN2k, Technische Universität Wien, Austria, 2001, ISBN 3-9501031-1-2.

[15] The LAPW/APW + lo basis set parameters are $R_{\mathrm{MT}}^{\mathrm{Cu}}=$ 2.2 bohr, $R_{\mathrm{MT}}^{\mathrm{Ag}}=2.4 \mathrm{bohr}, \quad R_{\mathrm{MT}}^{\mathrm{O}}=1.0 \mathrm{bohr}, \quad R_{\mathrm{MT}}^{\mathrm{C}}=$ $1.0 \mathrm{bohr}, E_{\mathrm{wf}}^{\max }=16 \mathrm{Ry}$, and $E_{\mathrm{pot}}^{\max }=400 \mathrm{Ry}$. A $(4 \times 4 \times$ 1) Monkhorst-Pack grid was used for reciprocal space sampling of the $(3 \times 3)$ surface unit cells.

[16] M.J. Frisch et al., GAUSSIAN03, revision C.02, Gaussian, Inc., Wallingford, CT, 2004.

[17] The $\mathrm{Cu}$ and $\mathrm{Ag}$ calculations employ a triple- $\zeta$ TZVP and a double- $\zeta$ LanL2DZ basis set, respectively. Extensive test calculations for $\mathrm{Cu}$ gave essentially identical results for 6-31+G, LanL2DZ, 6-311+G, and TZVP basis sets.

[18] The experimental CO total energy is obtained from the sum of all experimental ionization energies of the $\mathrm{O}$ and of $\mathrm{C}$ atoms (http://www.webelements.com/) and adding the experimental C-O binding energy of $11.109 \mathrm{eV}$ (see [24], which includes a zero point energy of $0.13 \mathrm{eV}$ ).

[19] W. Kohn, Phys. Rev. Lett. 76, 3168 (1996).

[20] K. Doll, Surf. Sci. 573, 464 (2004).

[21] M. Neef and K. Doll, Surf. Sci. 600, 1085 (2006).

[22] D. Spanjaard and M.C. Desjonqueres, in Interaction of Atoms and Molecules with Surfaces, edited by V. Bortolani, N.H. March, and N.P. Tosi (Plenum, New York, 1990), p. 255.

[23] M. Scheffler and C. Stampfl, in Handbook of Surface Science, edited by K. Horn and M. Scheffler (Elsevier, Amsterdam, 2000), Vol. 2, p. 286.

[24] D. R. Stull and H. Prophet, JANAF Thermochemical Tables, NBS, National Standards Reference Data Series - 37 (U.S. GPO, Washington, DC, 1971), 2nd ed.

[25] M. Fuchs, K. Reuter, and M. Scheffler (to be published).

[26] C. Filippi et al., Phys. Rev. Lett. 89, 166102 (2002).

[27] C. Tuma and J. Sauer, Chem. Phys. Lett. 387, 388 (2004); Phys. Chem. Chem. Phys. 8, 3955 (2006). 